\title{
INCREMENTO EN LA VIDA ÚTIL POST COSECHA DEL AGUACATE (Persea americana) UTILIZANDO RECUBRIMIENTOS A BASE DE GOMA GELANA
}

\section{POST-HARVEST SHELF-LIFE INCREASE OF AVOCADOS (Persea americana) USING GELLAN GUM BASED COATING}

\author{
Rafael González Cuello ${ }^{1}$, Jaime Pérez Mendoza² ${ }^{2}$ Víctor Gelvez Ordóñez ${ }^{3}$
}

\begin{abstract}
${ }^{1}$ Ph.D. Ingeniería de Alimentos, Docente Asistente. Universidad de Cartagena, Avenida del Consulado Calle 30 No. 48-152, Cartagena-Bolívar, Colombia, e-mail: rgonzalezc1@unicartagena.edu.co; ${ }^{2}$ MSc. Ingeniería de Alimentos, Docente Asociado. Universidad de Cartagena, Avenida del Consulado Calle 30 No. 48-152, Cartagena-Bolívar, Colombia, e-mail: jperezm@ unicartagena.edu.co; ${ }^{3}$ Ph.D. Ingeniería de Alimentos, Docente. Universidad de Pamplona, Colombia, e-mail: vmgelvez@ unipamplona.edu.co
\end{abstract}

Rev. U.D.CA Act. \& Div. Cient. 20(1): 101-110, Enero-Junio, 2017

\section{RESUMEN}

El aguacate es uno de los frutos de importancia económica en Colombia que presenta pérdidas en post cosecha. El objetivo de esta investigación fue evaluar el efecto de un recubrimiento comestible a base de gelana de alto (GAA) y bajo acilo (GBA), sobre la calidad post cosecha del aguacate (Persea americana cv. "Hass"), almacenado durante nueve semanas. Para la elaboración de los recubrimientos, se utilizó un diseño factorial $2^{3}$, donde los factores fueron las concentraciones de GAA $(0,30$ y $0,60 \%$ p/v), GBA $(0,30$ y $0,60 \% \mathrm{p} / \mathrm{v})$ y glicerol (GLI) $(8,0$ y $12 \% \mathrm{v} / \mathrm{v})$; como agente antimicrobiano, se utilizó extracto acuoso de Toronjil (Melissa officinalis L), por ser un aditivo natural. Se evaluaron propiedades, como la firmeza, la pérdida de humedad, el $\mathrm{pH}$ y los sólidos solubles totales (SST), durante almacenamiento. Finalmente, para la estimación del periodo de vida útil, se empleó la ecuación de Monod Hinshelwood, previo modelado de los datos, con ayuda de la ecuación de Baranyi y Roberts. Los resultados indicaron que la aplicación de recubrimientos comestibles de goma gelana, conteniendo bajas concentraciones de glicerol, disminuye significativamente $(P<0,05)$ la pérdida de firmeza, de humedad, de sólidos solubles y evitan grandes modificaciones del $\mathrm{pH}$, manteniendo la calidad del aguacate, alargando la vida útil.

Palabras clave: Conservación, extracto de toronjil, heteropolisacáridos, Persea americana, revestimientos comestibles.

\section{SUMMARY}

Avocado is one of the fruits of economic importance in Colombia. However, it presents losses mainly during the post- harvest period. The aim of this research was to evaluate the effect of an edible coating based on high acyl (HAG) and low acyl gellan (LAG) on post-harvest avocado quality (Persea americana $c v$. "Hass") stored during nine weeks. A factorial design $2^{3}$ was employed for the biofilm preparation, where the factors were concentrations of HAG $(0.30$ and $0.60 \% \mathrm{w} / \mathrm{v})$, LAG $(0.30$ and $0.60 \% \mathrm{w} / \mathrm{v})$ and glycerol $(\mathrm{GLY} \mathrm{v} / \mathrm{v})$. As antimicrobial aqueous extract of lemon balm (Melissa officinalis $L$ ) was used. Properties such as firmness, moisture loss, $\mathrm{pH}$, total soluble solids (TSS) during storage were evaluated. Finally, for the microbiological shelf life estimation the Hinshelwood Monod equation was applied previous data modeling using the Baranyi and Roberts model. The results indicated that the application of edible coatings based on gellan gum containing low concentrations of glycerol reduces the loss of firmness, moisture, soluble solids and prevent large changes in $\mathrm{pH}$, maintaining the quality of avocado and extend its shelf life.

Key words: Avocado, lemon balm, gellan gum, edible coating, post-harvest shelf life.

\section{INTRODUCCIÓN}

El empacado de matrices alimentarias se ha enfocado, principalmente, en el desarrollo de películas o recubrimientos biodegradables, elaboradas a partir de polisacáridos de origen vegetal (Seydim \& Sarikus, 2006) y microbiano, ya que los consumidores, actualmente, demandan una disminución en el uso de aditivos químicos, sobre las matrices alimentarias.

Los materiales empleados para la producción de recubrimientos, usualmente, son proteínas, polisacáridos y lípidos 
(Persin et al. 2011; Rodríguez \& Schobitz, 2009; González et al. 2015). Investigaciones previas han mostrado que varios polisacáridos, usados como agentes espesantes o estabilizantes, pueden también ser utilizados para la formación de recubrimientos (González et al. 2016).

La goma gelana es un heteropolisacárido lineal aniónico, producido por la bacteria Sphingomonas paucimobilis y consiste en unidades de repetición de un tetrasacárido (1,3- $\beta$-D-glucosa; 1,4- $\beta$-D-ácido glucurónico; 1,4 $\beta$-D glucosa; and 1,4- $\alpha$-L-ramnosa). La gelana nativa es conocida como gelana de alto acilo, debido a que presenta un grupo acetato (C6) y un grupo glicerato (C2), en su residuo de glucosa. Cuando la gelana de alto acilo es sometida a un fuerte tratamiento con álcali a elevadas temperaturas, se obtiene la gelana de bajo acilo. Esta diferencia estructural hace posible obtener geles de diferentes texturas (Buldo et al. 2016).

La capacidad de las películas y de los recubrimientos para retener humedad, aromas y transporte de solutos, puede ser mejorada, mediante la inclusión de aditivos, como antioxidantes, antimicrobianos, colorantes, saborizantes, nutrientes y especies, en la formulación (Pranoto et al. 2005). La influencia de los aditivos dependerá de su concentración, estructura química, grado de dispersión en el recubrimiento, así como su grado de interacción con el polímero. A pesar de los progresos realizados en el área de conservación de frutas y verduras, más de un cuarto de las frutas y vegetales cosechados se echan a perder, debido a su deterioro durante el almacenamiento (Maftoonazad \& Ramaswamy, 2005).

El aguacate (Persea americana Mill) es la quinta fruta tropical de mayor relevancia en el mundo, en términos de volumen y de área cultivada. Colombia, al producir más de 40 mil toneladas/año de aguacate hace que esta fruta tenga gran potencial para exportación; sin embargo, como consecuencia de una elevada demanda interna y una ausencia de adecuadas prácticas agrícolas, hacen difícil el acceso de esta fruta a mercados internacionales (Yabrudy, 2012).

El aguacate (Persea americana cv. "Hass") es una fruta con un excelente sabor y textura, que tiene un papel importante en la nutrición humana, por su contenido en ácidos, como el oleico, palmítico, linoleico, palmitoleico y esteárico, aparte de vitaminas, como la A, B, C, E, K y de un elevado contenido en fibra (Bill et al. 2014). Todo esto le confiere a la fruta efectos saludables, en la prevención del cáncer y enfermedades cardiovasculares (Awad \& Fink, 2000; Plaza et al. 2009). Infortunadamente, la vida útil del aguacate está condicionada por muchos factores, entre los cuales, es importante destacar el crecimiento de hongos (Elez et al. 2005), ya que las pérdidas post-cosecha por antracnosis pueden llegar a ser del $80 \%$, si no se emplean las medidas adecuadas (Bosse et al. 2013). En tal sentido, el uso de extractos acuosos ve- getales han sido utilizados, con el fin de incrementar la vida útil de matrices alimentarias (González et al. 2015). M. officinalis, también conocida como bálsamo de limón, bálsamo común o bálsamo dulce, es una hierba perenne, de olor a limón, que pertenece a la familia de la Lamiaceae y, a la cual, numerosos estudios le atribuyen una variada actividad biológica (Shakeri et al. 2016).

La vida útil del aguacate resulta ser de tres a cuatro semanas, cuando es almacenado en condiciones de humedad y temperaturas adecuadas. Varias técnicas han sido utilizadas, con el fin de extender la vida útil del aguacate, aunque algunas pueden presentar inconvenientes; por ejemplo, la refrigeración, si bien retrasa la maduración y prolonga la vida útil, el frío prolongado conlleva a una descomposición y daño del fruto (Ma et al. 2017).

En los aguacates, se ha estudiado la estabilidad oxidativa (Aguiló et al. 2014), la vida útil post cosecha (Maftoonazad \& Ramaswamy, 2005), la inducción de resistencia contra la antracnosis por aplicación combinada de aceite de tomillo y recubrimientos comestibles (Bill et al. 2014). Recientemente, se evaluó la actividad antagónica de diversos aislados bacterianos, obtenidos de múltiples fuentes contra bacterias fitopatógenas, aisladas de plantas de aguacate (Dunlap et al. 2016); sin embargo, hasta la fecha, no ha sido reportado el uso de mezclas binarias de gelana de alto y bajo acilo sobre aguacates. Por tal motivo, el objetivo de la presente investigación fue evaluar el efecto de un recubrimiento comestible binario, sobre la calidad pos cosecha del aguacate (P. americana).

\section{MATERIALES Y MÉTODOS}

Materiales. 45 frutos de tamaño uniforme de $P$. americana cu. "Hass" fueron obtenidos en el mercado local de la ciudad de Cartagena D.T. y C. (Colombia). Se seleccionaron, teniendo en cuenta su tamaño, el estado de madurez, la ausencia visual de deterioro ocasionado por microorganismos y daños en la piel. Los frutos fueron desinfectados con una solución de hipoclorito de sodio $(0,2 \mathrm{~g} / \mathrm{L})$ por 5 min y secados a temperatura ambiente (González et al. 2005) y luego divididos en dos lotes. El primer lote constituyó el grupo control, que se almacenó sin recubrimiento y, el segundo fue sometido a los diferentes tratamientos de recubrimientos con gelana.

Preparación de las biopelículas. Para la obtención de biopelículas, un diseño factorial $2^{3}$ fue utilizado, donde los factores fueron las concentraciones de gelana de alto acilo (GAA), (0,3 y 0,6\% p/p) y bajo acilo (GBA), (0,3 y 0,6\% p/p) y plastificante $(8,0$ y $12 \% \mathrm{v} / \mathrm{v})$, (Tabla 1$)$. Los polímeros fueron disueltos en agua destilada, con ayuda de una plancha de calentamiento, bajo agitación constante; luego, se incorporó glicerol (GLI), como agente plastificante. La solución resul- 
tante fue sometida a calentamiento $90^{\circ} \mathrm{C} / 10$ minutos; subsiguientemente, la temperatura fue disminuida, hasta alcanzar $30^{\circ} \mathrm{C}$, con el fin de adicionar el extracto acuoso de toronjil, obtenido mediante el procedimiento descrito por Herrera \& García (2006). Cada fruta fue pesada en una balanza analítica antes de ser recubierta. Los aguacates fueron sumergidos en la solución del recubrimiento durante $1 \mathrm{~min}$, a $20^{\circ} \mathrm{C}$; posteriormente, las frutas tratadas fueron secadas, para fijar el recubrimiento en la superficie de la fruta y, finalmente, las frutas fueron almacenadas junto con las muestras control durante nueve semanas. Los siguientes análisis fueron llevados a cabo para evaluar los cambios en la calidad de las muestras cada dos días, durante el periodo de almacenamiento (nueve semanas).

Tabla 1. Matriz experimental utilizada en la elaboración de las biopelículas.

\begin{tabular}{|c|c|c|c|}
\hline Tratamiento & GAA (\% p/v) & GBA (\% p/v) & GLI \%(v/v) \\
\hline 1 & 0,30 & 0,30 & 8,0 \\
\hline 2 & 0,30 & 0,60 & 12,0 \\
\hline 3 & 0,60 & 0,30 & 8,0 \\
\hline 4 & 0,60 & 0,60 & 12,0 \\
\hline 5 & 0,30 & 0,30 & 12,0 \\
\hline 6 & 0,30 & 0,60 & 8,0 \\
\hline 7 & 0,60 & 0,30 & 12,0 \\
\hline 8 & 0,60 & 0,60 & 8,0 \\
\hline
\end{tabular}

La pérdida de humedad fue estimada con ayuda de una balanza analítica. El contenido de sólidos solubles totales (SST) fue determinado con un refractómetro (Fischer, Extech Model 2132, Boston, EEUU). La firmeza fue medida mediante un texturometro (Shimadzu modelo EZ-Test EZ-S, Tokyo, Japón). Las muestras fueron sometidas a test de punción, a velocidad constante $40 \mathrm{~mm} / \mathrm{mm}$, utilizando una geometría de punta redonda de $5 \mathrm{~mm}$ de diámetro. Curvas de fuerza deformación fueron realizadas, con el fin de obtener la pendiente $(\mathrm{N} / \mathrm{mm})$ de la zona lineal que representa la firmeza. $\mathrm{Al}$ menos diez determinaciones fueron llevadas a cabo en cada fruta, reportando los promedios aritméticos. Los valores de $\mathrm{pH}$ fueron obtenidos usando un $\mathrm{pH}$ metro (Thompson Bante Instrument, Shanghai, China). Una maceración fue realizada con una muestra de aguacate en agua destilada $(1: 10 \mathrm{p} / \mathrm{v})$, previa agitación durante $10 \mathrm{~min}$.

Estimación de la vida útil microbiológica del aguacate. La estimación de la vida útil fue llevada a cabo, tanto para las muestras control como para las recubiertas, realizando varias cinéticas de crecimiento, a distintas temperaturas (10 $-25^{\circ} \mathrm{C}$ ), utilizando como microorganismos indicadores, los mohos y las levaduras en general, sin considerar un género en particular. Los datos obtenidos fueron modelados matemáticamente, utilizando la ecuación de Baranyi y Roberts (1994) (Ecuación 1), por medio del programa de cómputo DMFit 2.0:

$$
\begin{gathered}
y(t)= \\
y_{0}+\mu_{\max } t+\frac{1}{\mu_{\max }} \ln \left(e^{-v t}+e^{-\mathrm{h}_{0}}-e^{\left.-v t-\mathrm{h}_{0}\right)}-\right. \\
\frac{1}{m} \ln \left[1+\frac{e^{m \mu_{\max } t+\frac{1}{\mu_{\max }} \ln \left(e^{-v t_{+}} e^{-\mathrm{h}_{0}-e^{\left.-v t-\mathrm{h}_{0}\right)}}-1\right.}}{e^{m\left(y_{\max }-y_{0}\right)}}\right]
\end{gathered}
$$

(Ec.1)

Donde: $y(t)$ es la concentración celular o el diámetro de las colonias; yo es la concentración o diámetro inicial; $\mu$ máx es la velocidad específica de crecimiento (1 $h$ ); m es un parámetro de curvatura para caracterizar la transición de la fase exponencial; $v$ es un parámetro de curvatura para caracterizar la transición a la fase exponencial y ho es un parámetro adimensional que cuantifica el estado fisiológico inicial de las células. Una vez obtenidos los parámetros cinéticos de crecimiento microbiano, se procedió a estimar la vida útil microbiológica, utilizando la ecuación de Monod-Hinshelwood (ecuación 2):

$$
t s=\frac{\log N s-\log N o}{\log 2} X T d
$$


Donde: ts es el tiempo necesario para que se desarrolle la alteración en el alimento; Ns (ufc/g) es el valor correspondiente a la población de seguridad (valor máximo permisible antes de considerarse alterado el producto); No (ufc/g) es el valor correspondiente a la población inicial presente en el producto, que es estimado mediante recuento del microorganismo por siembra en placa profunda al inicio de la cinética microbiana; Td es el tiempo de duplicación promedio, obtenido de las cinéticas microbianas a las diferentes temperaturas $\left(10-25^{\circ} \mathrm{C}\right)$. Es importante mencionar que Td es el cociente de In2 y la velocidad específica de crecimiento microbiano y esta última corresponde a la pendiente de la fase logarítmica de crecimiento del microorganismo.

Análisis de datos. Los datos obtenidos fueron analizados mediante análisis normal de varianza (ANOVA un factor), empleando la prueba de Tukey, con un nivel de confianza del 95\%, utilizando el programa de computo SPSS (Statistical Package for the Social Sciences Inc. Chicago, IL, USA) versión 17.0 para Windows.

\section{RESULTADOS Y DISCUSIÓN}

Pérdida de humedad. En la figura 1, se presentan los cambios en la humedad del aguacate en función del tiempo de almacenamiento, en donde se puede apreciar que el porcentaje de pérdida de humedad en las muestras control (sin recubrimiento) fue significativamente $(P<0,05)$ mayor en comparación a las muestras recubiertas. En las frutas control, la pérdida de humedad promedio fue aproximadamente de 9,66 durante las nueve semanas de almacenamiento, mientras que las frutas recubiertas con la proporción 0,6GBA/0,6GAA/8,0GLI presentaron la menor pérdida de peso, con $1,10 \%$, durante el mismo periodo. Las frutas que fueron recubiertas utilizando una proporción del 12\% (v/v) de GLI, independientemente de las concentraciones de gelana utilizadas, presentaron valores de pérdida de humedad entre 3,58 y 4,55\%; por el contrario, las frutas recubiertas con menores concentraciones de GLI $(8,0 \% \mathrm{v} / \mathrm{v})$, sin importar las proporciones de gelana, presentaron menores porcentajes de pérdida de humedad (1,10 - 1,91\%). Estos resultados son similares a los reportados por Jeong et al. (2003), en frutas tratadas con mezclas de cera y metil ciclopropeno, donde encontraron valores entre 1,90 y 5,4, dependiendo de las concentraciones de cera utilizadas. Igualmente, Saucedo et al. (2009) reportaron valores de pérdida de humedad cercanos al 5,0\%, después de seis semanas de almacenamiento, en aguacates recubiertos con cera candelilla, conteniendo ácido elágico. Los menores valores de pérdida de humedad obtenidos en las frutas recubiertas pueden ser atribuidos a las propiedades de barrera para la difusión de gases de las estomas, organelos que regulan el proceso de transpiración, además del intercambio de gases, entre la fruta y el medio ambiente (Salunke et al. 1991).
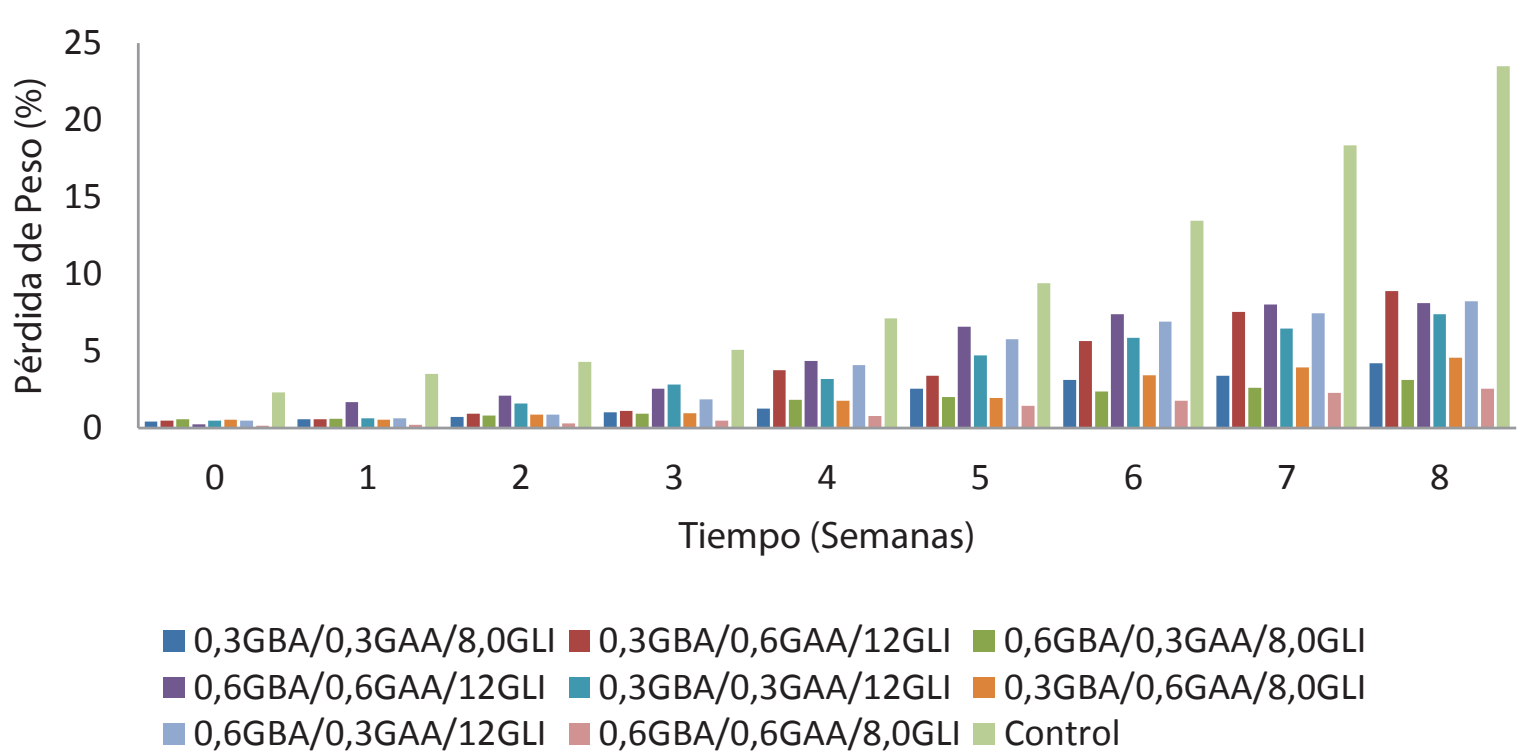

Figura 1. Pérdida de humedad de frutas de aguacate recubiertas a distintas proporciones de gelana de alto (GAA), bajo acilo (GBA) y glicerol (GLI). 
El mecanismo de pérdida de humedad en frutas y en vegetales también puede ser ocasionado por la diferencia en el gradiente de presión de vapor de agua, entre la parte interna y externa del fruto, por lo cual, los recubrimientos ayudan a reducir la pérdida de humedad, debido a la formación de una película en la superficie de la fruta. El espesor de la película, así como la permeabilidad del recubrimiento son aspectos importantes, ya que afectan la velocidad de transferencia de masa y, por tanto, deberán estudiarse en un futuro.

Contenido de sólidos solubles totales (SST). En la figura 2 , se puede observar el incremento significativo $(P<0,05)$ en la concentración de SST, en las muestras con recubrimien- to, durante las 9 semanas de almacenamiento, alcanzando concentraciones finales, entre 1,76 y $3,54^{\circ}$ Brix; es importante aclarar que todos los frutos presentaron concentraciones iguales (1,21 - 1,22 Brix) al inicio del almacenamiento (tiempo cero). El contenido de sólidos fue en promedio mayor en aguacates sin recubrimiento $\left(3,02^{\circ}\right.$ Brix), en comparación con las muestras con recubrimiento; posiblemente, este comportamiento es relacionado al proceso de maduración acelerado de las frutas control y la correspondiente pérdida de peso en la misma $(\mathrm{P}<0,05)$ (Figura 1$)$; en este caso, los componentes volátiles y aquellos solubles en agua, pueden ser liberados al entorno, durante la deshidratación de las muestras (Saucedo et al. 2009).
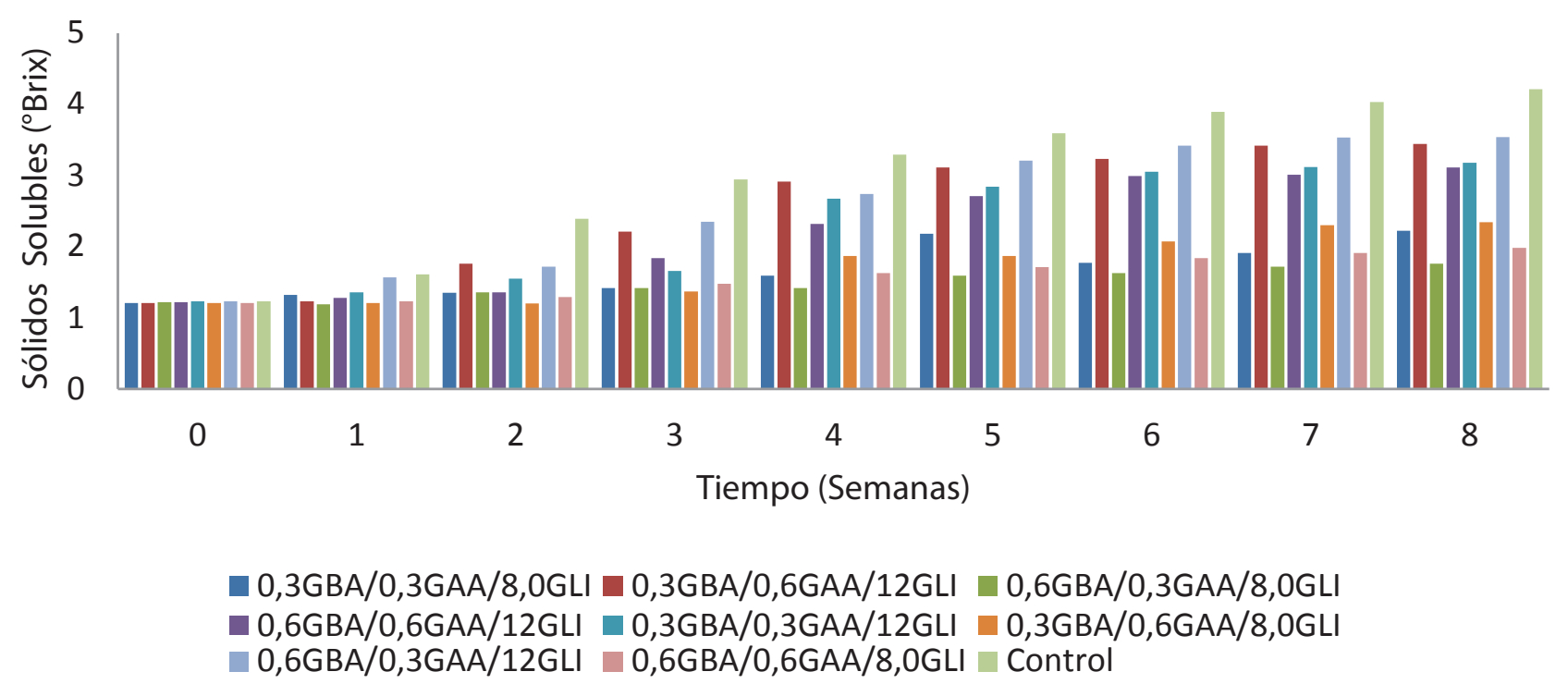

Figura 2. Contenido de sólidos solubles en frutas de aguacate recubiertas a distintas proporciones de gelana de alto (GAA), bajo acilo (GBA) y glicerol (GLI).

El comportamiento de las muestras recubiertas presenta dos tendencias con respecto el contenido de GLI: los recubrimientos con 8,0\% (v/v) mostraron concentraciones de SST, de 1,47 a $1,71^{\circ}$ Brix, mientras que los recubrimientos con una mayor concentración de plastificante $(12 \% \mathrm{v} / \mathrm{v})$ presentaron mayores valores, entre 2,20 y 2,59 ${ }^{\circ}$ Brix. Este comportamiento coloca en evidencia un mayor efecto de las concentraciones de plastificante en comparación a las proporciones de GAA y GBA.

Valores de $\mathrm{pH}$. Los valores de $\mathrm{pH}$ se incrementaron durante el periodo de estudio, para todas las muestras de aguacate analizadas (Figura 3), debido al consumo de moléculas orgánicas en los ciclos metabólicos, que proporcionan la ener- gía que el fruto requiere; además, algunos ácidos orgánicos participan como precursores de sustancias volátiles (Park et al. 2006). La muestra que presentó los valores más elevados de $\mathrm{pH}$ fue la de sin recubrimiento $(7,38)$, mientras que con la muestra recubierta de $0,3 \mathrm{GBA} / 0,3 \mathrm{GAA} / 8,0 \mathrm{GLI}$, se obtuvieron los menos valores de $\mathrm{pH}(6,40)$. Estos resultados son comparables con los obtenidos por Márquez et al. (2014), quienes reportaron incrementos en los valores de $\mathrm{pH}$, desde 6,42 hasta 6,63, en muestras de aguacate (Persea americana Mill. cv. "Hass"), almacenados durante 21 días. Igualmente, González et al. (2005) encontraron un incremento de $\mathrm{pH}$ de muestras de papaya tratada con recubrimientos, como una consecuencia natural del proceso de maduración. 


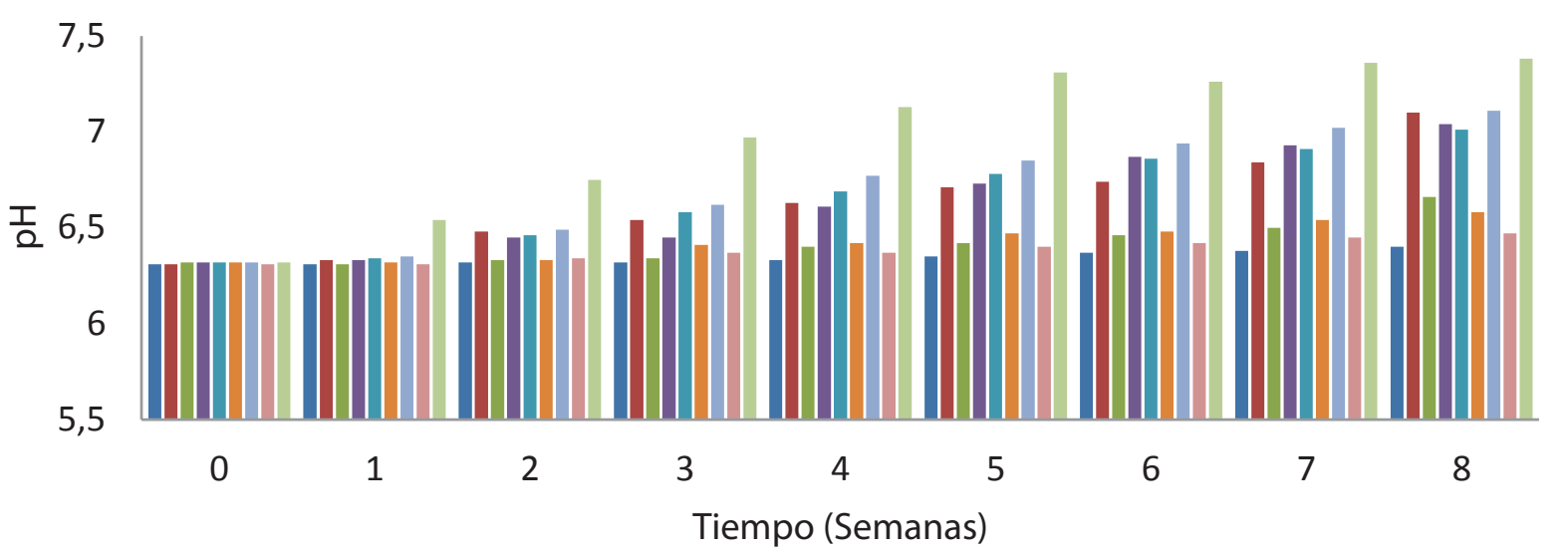

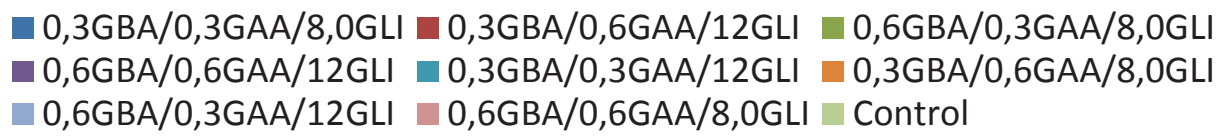

Figura 3. Valores de $\mathrm{pH}$ en frutas de aguacate recubiertas a distintas proporciones de gelana de alto (GAA), bajo acilo (GBA) y glicerol (GLI).

Es interesante mencionar que en algunas muestras de aguacate recubiertas con distintas proporciones de GAA y GBA, no se presentaron diferencias significativas $(P<0,05)$; sin embargo, al variar las concentraciones de GLI en los recubrimientos, sí se apreciaron diferencias $(P<0,05)$, es decir, los recubrimientos que contenían $8,0 \%(\mathrm{v} / \mathrm{v})$ de GLI presentaron niveles de $\mathrm{pH}$, entre 6,40 y 6,66, al finalizar el tiempo de almacenamiento (9 semanas), mientras que aguacates con recubrimiento conteniendo mayor concentración de GLI $(12 \% \mathrm{v} / \mathrm{v})$ mostraron mayores valores de $\mathrm{pH}(7,01-7,11)$; este comportamiento se aprecia claramente al observar la figura 3.

Firmeza. En la primera semana, se presentaron las mayores resistencias a la penetración, con valores de firmeza de 74,3 Newton (N), para los frutos sin recubrimiento y entre 73,7 y 75,8 $\mathrm{N}$, para frutos con recubrimiento. Los aguacates recubiertos y sin recubrir presentaron una disminución en los valores de firmeza, hasta 7,6 $\mathrm{N}$ y 3,5 $\mathrm{N}$, respectivamente, después de la 9 semana de almacenamiento; sin embargo, la aplicación de los recubrimientos mostró un efecto benéfico en la retención de la firmeza, ya que las frutas recubiertas lograban tener valores de resistencia, entre 20,1 y 45,7 N, después de seis semanas de almacenamiento; por el contrario, las frutas sin recubrimiento, solo lograban tener valores de resistencia de 13,6 N. En la figura 4, nuevamente se aprecia la mayor incidencia de las concentraciones de GLI en comparación a las proporciones de GBA y GAA. Este efecto es marcado después de la segunda y hasta la séptima semana de almacenamiento; durante este tiempo, se aprecian diferencias significativas $(P<0,05)$, en los valores de firmeza, obtenidos en muestras de aguacate con recubrimientos conteniendo GLI, en distintas proporciones (8,0 y 12\%v/v).

Jeong et al. (2003) encontraron que frutas sin recubrir se ablandan y maduran completamente dentro de 7 días de almacenamiento, a $20^{\circ} \mathrm{C}$. En contraste, frutas recubiertas con cera y metil ciplopropeno presentan, aproximadamente, una retención de la tercera parte de la firmeza después de siete días de almacenamiento, a $20^{\circ} \mathrm{C}$. La retención de la firmeza puede ser explicada por la degradación de la protopéctina insoluble a compuestos más solubles, como el ácido péctico. Durante la maduración de la fruta ocurre una despolimerización de las sustancias pécticas, con un incremento en la actividad enzimática (pectin- esterasa y poligalacturonasa) (Salunke et al. 1991; Maftoonazad \& Ramaswamy, 2005).

Vida útil Microbiológica. La vida útil microbiológica de un producto alimenticio, se puede estimar obteniendo los parámetros cinéticos de crecimiento microbiano, extraídos de la fase logarítmica, previo modelado de los datos, utilizando la ecuación de Baranyi \& Roberts (1994). Los valores obtenidos en la estimación de la vida útil microbiológica, se muestran en la tabla 2, en donde se puede apreciar que, el mayor tiempo de vida útil (52,2 días), se encontró en el aguacate recubierto con $0,6 \%(\mathrm{p} / \mathrm{v})$, de GAA; 0,6\% (p/v), de 

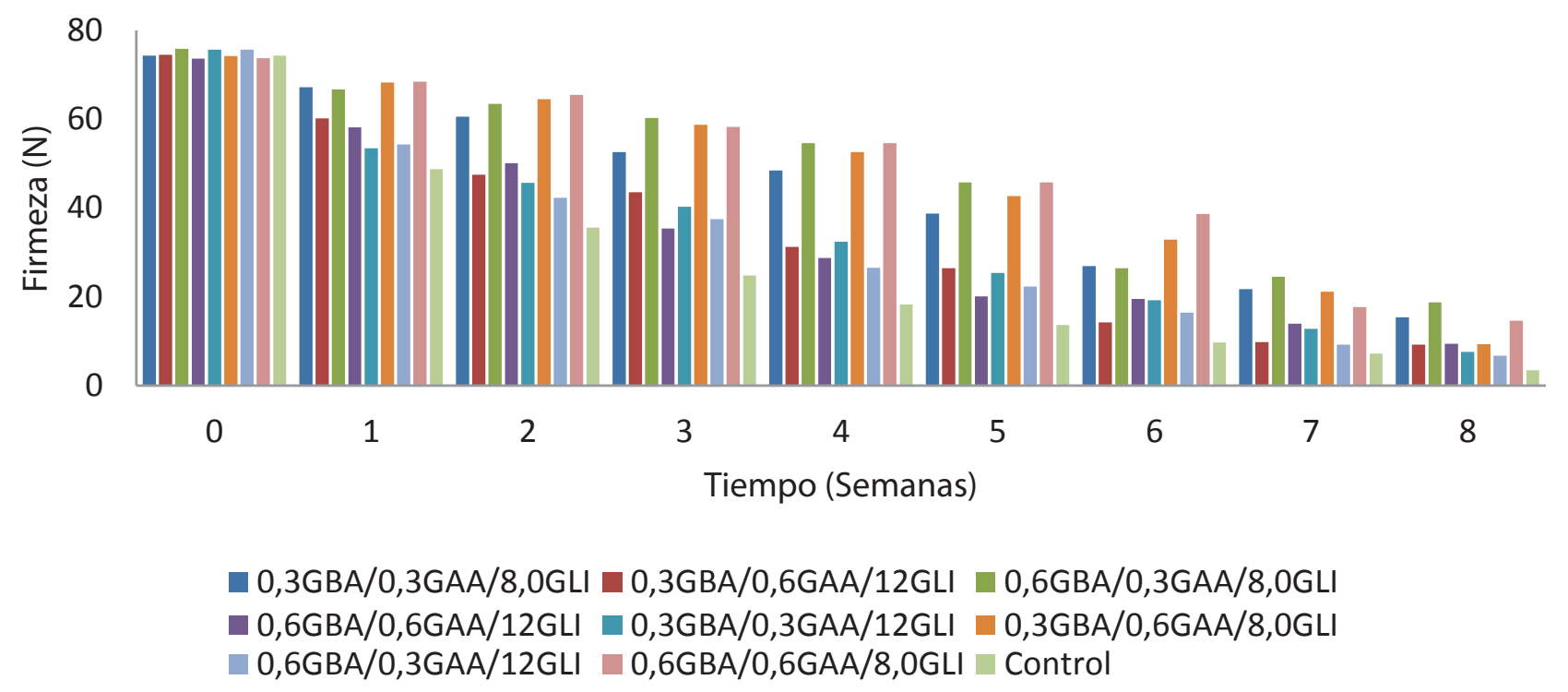

Figura 4. Firmeza de frutas de aguacate recubiertas a distintas proporciones de gelana de alto (GAA), bajo acilo (GBA) y glicerol (GLI).

GBA y $8,0 \%(v / v)$, de GLI, seguida por las combinaciones de $0,3 \%(\mathrm{p} / \mathrm{v})$, de GAA; $0,6 \%(\mathrm{p} / \mathrm{v})$, de GBA y $8,0 \%(\mathrm{v} / \mathrm{v})$, de GLI con 49,9 días.

En términos generales, las mayores vidas útiles $(46,4-52,2$ días), se encontraron en frutos, en los cuales, el recubrimiento contenía bajas concentraciones de GLI ( $8,0 \% \mathrm{v} / \mathrm{v})$; por el contrario, la menor vida útil, se registró en frutos sin recubrimien- to (control), con 35,8 días, seguida por las frutas, en las que el recubrimiento tenía $12 \%$ (v/v) de GLI, con vidas útil, entre 38,3 y 42,7 días. Estos resultados confirman el mayor efecto que presentan las concentraciones de GLI sobre los parámetros estudiados (SST, pH, firmeza, pérdida de humedad y vida útil), en comparación a las proporciones de GBA y GAA.

Es importante destacar que el aumento en la vida útil de

Tabla 2. Valores de estimación de la vida útil de aguacate recubiertas a distintas proporciones de gelana de alto (GAA), bajo acilo (GBA) y glicerol (GLI).

\begin{tabular}{|c|c|c|c|c|c|}
\hline GAA (\% p/v) & GBA (\% p/v) & GLI \%(v/v) & Ecuación & $\mathbf{R}^{2}$ & Días \\
\hline 0,30 & 0,30 & 8,0 & $\log _{10} T d=-0,092 x+2,273$ & 0,976 & 46,4 \\
\hline 0,30 & 0,60 & 12,0 & $\log _{10} T d=-0,087 x+2,210$ & 0,965 & 42,7 \\
\hline 0,60 & 0,30 & 8,0 & $\log _{10} T d=-0,0594 x+2,152$ & 0,945 & 48,5 \\
\hline 0,60 & 0,60 & 12,0 & $\log _{10} T d=-0,0842 x+2,213$ & 0,999 & 43,2 \\
\hline 0,30 & 0,30 & 12,0 & $\log _{10} 10 T d=-0,069 x+2,098$ & 0,956 & 38,3 \\
\hline 0,30 & 0,60 & 8,0 & $\log _{10} T d=-0,0681 x+2,183$ & 0,988 & 49,9 \\
\hline 0,60 & 0,30 & 12,0 & $\log _{10} T d=-0,0820 x+2,174$ & 0,997 & 40,6 \\
\hline 0,60 & 0,60 & 8,0 & $\log _{10} T d=-0,0712 x+2,241$ & 0,972 & 52,2 \\
\hline Control & & & $\log _{10} T d=-0,0538 x+2,008$ & 0,999 & 35,8 \\
\hline
\end{tabular}


los aguacates puede ser atribuido al efecto antimicrobiano ejercido por el extracto acuoso de toronjil, que limita el crecimiento de microorganismos en la superficie de la matriz alimentaria; este efecto, se debe a los compuestos presentes en las hojas de toronjil (Montes, 2009; Acevedo et al. 2013).

Compuestos extraídos de materiales vegetales inhiben el crecimiento de microorganismos patógenos y la germinación de esporas, porque afectan los sitios activos de las enzimas y el metabolismo celular (Arrebola et al. 2010). La presencia de anillos fenólicos y de grupos hidroxilos de los fenoles presentan actividad antimicrobiana (Bagamboula et al. 2004).

Los recubrimientos, dependiendo del principio activo que contengan, pueden inhibir la actividad enzimática de las poligalacturonasas, pectatoliasas y celulasas (Atkinson et al. 2012), las cuales, están involucradas en el proceso de maduración. Ghaouth et al. (1991) reportaron que el uso de recubrimientos comestibles ocasiona leves cambios en la apariencia de las manzanas; estos autores argumentan que dichos cambios, se deben a una modificación en la atmósfera creada en el fruto, con altos niveles de $\mathrm{CO}_{2}$ y bajas concentraciones de $\mathrm{O}_{2}$, conllevando a una disminución del proceso de maduración.

La presencia de grupos acilo y el consecuente impedimento estérico que ocasionan en la agregación de las cadenas poliméricas de goma gelana, no incide en ninguna de las características (SST, pH, firmeza, pérdida de humedad y vida útil), evaluadas en el presente estudio; por el contrario, las concentraciones de GLI, sí ocasionan modificaciones en las mencionadas características. Este comportamiento puede ser generado por la disminución de las fuerzas intermoleculares en las cadenas del polímero, que causa una mayor movilidad molecular, incrementando la permeabilidad al agua. Igualmente, los plastificantes incrementan el volumen libre, por ende, existe más espacio para la migración de las moléculas de agua. Los plastificantes hidrofilicos tipo GLI son compatibles con los materiales poliméricos que originan las biopelículas, aumentando la capacidad de sorción de moléculas polares, como el agua, otra razón para incrementar los valores de permeabilidad. Además, los grupos polares $(-\mathrm{OH})$, junto a las cadenas de plastificante, se cree que desarrollan enlaces polímero-plastificante, reemplazando las interacciones polímero-polímero en las películas de poliméricas (Yang \& Paulson, 2000).

La aplicación de recubrimientos comestibles de goma gelana y extracto de toronjil, conteniendo bajas concentraciones de glicerol disminuyen la pérdida de firmeza, de humedad, de sólidos solubles y evitan grandes modificaciones del $\mathrm{pH}$, manteniendo la calidad del aguacate, al extender la vida útil, comparada con las frutas control. Estos resultados sugieren la posibilidad de utilizar recubrimientos indistintos de goma gelana de alto y bajo acilo, a bajas concentraciones de glicerol, como una alternativa para ser empleadas en la conservación de frutas.

Conflictos de intereses: El manuscrito fue preparado y revisado con la participación de todos los autores, quienes declaramos que no existe conflicto de intereses que ponga en riesgo la validez de los resultados presentados. Financiación: Esta investigación fue llevada a cabo en la Universidad de Cartagena.

\section{BIBLIOGRAFÍA}

1. ACEVEDO, D.; NAVARRO, M.; MONTERO, M. 2013. Composición Química del Aceite Esencial de las Hojas de Toronjil (Melissa officinalis L.). Inf. Tecnol. 24(4):49-54.

2. AGUILÓ, A.; OMS, O.; MARTíN, B.; SOLIVA, F. 2014. Impact of pulsed light treatments on quality characteristics and oxidative stability of fresh-cut avocado. LWT - Food Sci. Technol. 59:320-326.

3. ARREBOLA, E.; SIVAKUMAR, D.; BACIGALUPO, R.; KORTSEN, L. 2010. Combined application of antagonist Bacillus amyloliquefaciens and essential oils for the control of peach postharvest diseases. Crop Prot. 29:369-377.

4. ATKINSON, R.; SUTHERLAND, P.; JOHNSTON, S.; GUNASEELAN, K.; HALLETT, I.; MITRA, D.; BRUMMELL, D.; SCHRODER, R.; JOHNSTON, J.; SCHAFFER, R. 2012. Down-regulation of polygalacturonase alters firmness, tensile strength and water loss in apple (Malus domestica) fruit. BMC Plant Biol. 12:129.

5. AWAD, A.; FINK, C. 2000. Phytosterols as anticancer dietary components: evidence and mechanism of action. J. Nutr. 130(9):2127-2130.

6. BAGAMBOULA, C.; UYTTENDAELE, M.; DEBEVERE, J. 2004. Inhibitory effect of thyme and basil essential oil, carvacol, thymol, estragol, linalool and p-cymene towards Shigella sonnei and S. flexneri. Food Microbiol. 21:33-42.

7. BARANYI, J.; ROBERTS, T. 1994. A dynamic approach to predicting bacterial growth in food. International J. Food Microbiol. 23(3-4):277-294.

8. BILL, M.; SIVAKUMAR, D.; KORSTEN, L.; THOMPSON, K. 2014. The efficacy of combined application of edible coatings and thyme oil in inducing resistan- 
ce components in avocado (Persea americana Mill.) against anthracnose during post-harvest storage. Crop Prot. 64:159-167.

9. BOSSE, R.; BOWER, J.; BERTLING, I. 2013. Systemic resistance inducers applied preharvest for Colletotrichum gloeosporioides control in avocados. Acta Hort. 1007:153-160.

10. BULDO, P.; BENFELDT, C.; CAREY, J.; FOLKENBERG, M.; JENSEN, B.; SIEUWERTS, S.; VLACHVEI, K.; IPSEN, R. 2016. Interactions of milk proteins with low and high acyl gellan: Effect on microstructure and textural properties of acidified milk. Food Hydrocol. 60: 225-231.

11. DUNLAP, C.; LUESCHOWA, S.; CARRILLO, D.; ROONEY, A. 2016. Screening of bacteria for antagonistic activity against phytopathogens of avocados. Plant Gene. En prensa: http://dx.doi.org/10.1016/j.plgene.2016.11.004

12. ELEZ, M.P.; SOLIVA, R.C.; GORINSTEIN, S.; MARTÍNBELLOSO, O. 2005. Natural antioxidants preserve the lipid oxidative stability of minimally processed avocado puree. J. Food Sci. 70(5):S325-S329.

13. GHAOUTH, E.; ARUL, J.; PONNAMPALAM, R. 1991. Use of chitosan coating to reduce water loss and maintain quality of cucumber and bell pepper fruits. J. Food Proc. Pres. 15:359-368.

14. GONZÁLEZ, G.; MONROY, I.; GOYCOOLEA, V.; DÍAZ, M.; AYALA, J. 2005. Cubiertas comestibles de quitosano. Una alternativa para prevenir el deterioro microbiano y conservar la calidad de papaya fresca cortada. In Proceedings. (p.121-133). La Habana, Cuba.

15. GONZÁLEZ-AGUILAR, G.A.; MONROY-GARCÍA, I.N.; GOYCOOLEA-VALENCIA, F.; DÍAZ-CINCO, M.E.; AYALA ZAVALA, J.F. 2005. Cubiertas comestibles de quitosano. Una alternativa para prevenir el deterioro microbiano y conservar la calidad de papaya fresca cortada. In: Proceedings of the Simposium Nuevas tecnologías de conservación y envasado de frutas y hortalizas Vegetales frescos cortados. La Habana, Cuba. p.121-133.

16. GONZÁLEZ, R.; CASTRO, B.; CARO, Y. 2015. Obtención de biopelículas binarias activas y su efecto en la vida útil microbiológica del camarón blanco (Penaeus vannamei). Inf. Tecnol. 26(5):3-10.
17. GONZÁlEZ R.; PATERNINA, L.; CARRILLO, A. 2016. Biopelículas terciarias: fuerza de ruptura y efecto sobre la vida útil de cortes de tilapia negra (Oreochromis niloticus). Inf. Tecnol. 27(1):33-40.

18. HERRERA, F.; GARCÍA, R. 2006. Evaluación in vitro del efecto bactericida de extracto acuso de laurel, clavo, canela y tomillo sobre cinco cepas bacterianas patógenas de origen alimentario. Rev. Bistua. 4(2):13-19.

19. JEONG, J.; HUBER, D.; SARGENT, S. 2003. Delay of avocado (Persea americana) fruit by 1-methylcyclopropene and wax treatments. Post. Biol. Technol. 28:247-257.

20. MA, L.; ZHANG, M.; BHANDARI, B.; GAO, Z. 2017. Recent developments in novel shelf life extension technologies of fresh-cut fruits and vegetables. Trends Food Sci \& Technol. En prensa: doi: 10.1016/j. tifs.2017.03.005.

21. MAFTOONAZAD, N.; RAMASWAMY, H. 2005. Postharvest shelf-life extension of avocados using methyl cellulose-based coating. LWT - Food Sci. Technol. 38:617-624.

22. MÁRQUEZ, C.; YEPES, D.; SÁNCHEZ, L.; OSORIO, J. 2014. Cambios físico-químicos del aguacate (Persea americana Mill. cv. "Hass") en poscosecha para dos municipios de Antioquia. Temas Agrarios. 19(1):3247.

23. MONTES, R.T. 2009. Diversidad de compuestos químicos producidos por las plantas contra hongos fitopatógenos: revisión. Rev. Mex. Micol. 29:74-82.

24. PARK, Y.; JUNG, S; GORINSTEIN, S. 2006. Ethylene treatment of 'Hayward' kiwifruits (Actinidia deliciosa) during ripening and its influence on ethylene biosynthesis and antioxidant activity. Scientia Horticulturae. 108: 22-28.

25. PERSIN, Z.; STANA, K.; FOSTER, T.; VAN DAM, J.; BOERIU, C.; NAVARD, P. 2011. Challenges and opportunities in polysaccharides research and technology: The EPNOE views for the next decade in the areas of materials, food and health care. Carb. Polym. 84(1):22-32.

26. PLAZA, L.; SÁNCHEZ. M.; DE PASCUAL, T.; DE ANCOS, B.; CANO, M. 2009. Fatty acids, sterols, and antioxidant activity in minimally processed avocados during refrigerated storage. J. Agricul. Food Chem. 57(8):3204-3209. 
27. PRANOTO, Y.; RAKSHIT, S.; SALOKHE, V. 2005. Enhancing antimicrobial activity of chitosan films by incorporating garlic oil, potassium sorbate and nisin. LWT- Food Sci. Technol. 38:859-865.

28. RODRÍGUEZ, R.; SCHOBITZ, R.E. 2009 Película antimicrobiana a base de proteína de suero lácteo, incorporada con bacterias lácticas como controlador de Listeria monocytogenes, aplicada sobre salmón ahumado. Biotecnol. Sec. Agrop. Agroind. 7(2):4954.

29. SALUNKE, D.; BOUN, H.; REDDY, N. 1991. Storage processing and nutritional quality of fruits and vegetables. Boston, MA. USA, CRC Press Inc. p.156-161.

30. SAUCEDO, S.; ROJAS, R.; AGUILERA, A.; SAENZ, G.; DE LA GARZA, E.; JASSO, D.; AGUILAR, C. 2009. Edible film based on candelilla wax to improve the shelf life and quality of avocado. Food Res. Int. 42: 511-515.
31. SHAKERI, A.; SAHEBKAR, A.; JAVADI, B. 2016. Melissa officinalis L. - A review of its traditional uses, phytochemistry and pharmacology. J. Ethnoph. 188:204228.

32. SEYDIM, A.; SARIKUS, G. 2006. Antimicrobial activity of whey protein based edible films incorporated with oregano, rosemary and garlic essential oils. Food Res. Int. 39:639-644.

33. YANG, L.; PAULSON, A. 2000. Mechanical and water vapor barrier properties of edible gellan films. Food Res. Int. 33(7):563-570.

34. YABRUDY, J. 2012. El Aguacate en Colombia: Estudio de caso de los Montes de María, en el Caribe colombiano. Banco de la República - Cartagena. 171:615.

Recibido: Diciembre 15 de 2016

Aceptado: Abril 24 de 2017

Cómo citar:

González Cuello, R.; Pérez Mendoza, J.; Gelvez Ordóñez, V. 2017. Incremento en la vida útil post cosecha del aguacate utilizando recubrimientos a base de goma gelana. Rev. U.D.C.A Act. \& Div. Cient. 20(1): 101-110. 